\title{
Forensic linguistics. Reality Monitoring and Criteria Based Content Analysis (CBCA): comparison between methods
}

\section{Linguistica forense. Reality Monitoring e Criteria Based Content Analysis (CBCA): metodi a confronto}

\section{Linguistica forense. Reality Monitoring y Criteria Based Content Analysis (CBCA): comparación de metodologías}

\author{
Vincenzo M. Mastronardi, ${ }^{1}$ Monica De Vincentis ${ }^{2}$ \\ ${ }^{1}$ Psichiatra Criminologo clinico Docente a.c. Sapienza Università di Roma e Università degli Studi Internazionali di Roma; \\ ${ }^{2}$ Laureanda in Investigazione, Criminalità e Sicurezza internazionale, Università degli Studi Internazionali di Roma, Italy
}

\begin{abstract}
Forensic linguistics is about the analysis of written, oral or non-verbal language in the legal field. For this purpose it employs tools finalized to the quantification and qualification of information. Among those we find Reality Monitoring, that discriminates between internal and external memories; Criteria Based Content Analysis, which uses nineteen criteria to verify the authenticity of a speech; Statement Validity Analysis; Scientific Content Analysis; Step Wise Interview. The comparison between these disciplines (and an overview on those) is preceded by important observations about cognitive and mnemonic processes and the role of the interviewer in the collection of testimony.
\end{abstract}

\section{RIASSUNTO}

La linguistica forense si occupa dell'analisi del linguaggio, scritto, orale e non verbale, in ambito legale. Si avvale, a questo scopo, di strumenti per quantificare e qualificare le informazioni esaminate, tra cui il Reality Monitoring, utilizzato per discriminare memorie interne ed esterne; la Criteria Based Content Analysis, che permette, attraverso diciannove criteri, di verificare la veridicità di un racconto; la Statement Validity Analysis; la Scientific Content Analysis; la Step Wise Interview. Il confronto tra queste discipline (ed una panoramica sulle stesse) è preceduto da importanti osservazioni circa i processi cognitivi e mnemonici ed il ruolo dell'operatore nelle interviste finalizzate alla testimonianza.

\section{RESUMEN}

La linguistica forense se ocupa del análisis de la lengua, escrita, oral y no-verbal, de dictado legal. A este respecto, hace uso de instrumentos para cuantificar y cualificar a las informaciones examinadas, por ejemplo el Reality Monitoring, utilizado para discriminar entre recuerdos internos y externos; la Criteria Based Content Analysis que, a través de diecinueve criterios, permite verificar la veracidad de un cuento; la Statement Validity Analysis; la Scientific Content Analysis; la Step Wise Interview. La comparación entre esas disciplinas (y una visión general sobre las mismas) esta adelantado por importantes observaciones sobre los procesos cognitivos y mnemonicos y el papel del profesional en las entrevistas para el testimonio.

\section{La linguistica forense}

La linguistica forense è una disciplina che si occupa dell'analisi di elementi legati al linguaggio in ambito legale, avvalendosi di applicazioni di stilistica, analisi del discorso, neurolinguistica, psico-linguistica (etc.), e trovando realizzazione nell'osservazione di espressioni scritte, verbali e non verbali. Lo studio del linguaggio risponde sostanzialmente a tre interrogativi (Mastronardi, 2014):

- “cosa c'è scritto?": è importante, come passaggio primario, decifrare il contenuto linguistico che ci si appresta ad analizzare. Ciò si applica non solo per il discorso scritto, ma anche per quanto riguarda testimonianze orali;

- “cosa significa?": il significato attribuito a linguaggio scritto, orale o non verbale è fondamentale per comprendere il messaggio che il contenuto intende comunicare;
- “chi è l'autore?": attraverso l'identificazione dell'autore è possibile dare un volto ed un contesto al linguaggio o, al contrario, utilizzare una determinata espressività linguistica (idioletto) per determinare età, sesso e caratteristiche psicologiche del mittente.

La disciplina si avvale, inoltre, del principio di identificazione (Huber, 1959; Osborn, 1946; Trojani, 1998): “Quando due enti hanno caratteristiche comuni, che per qualità e quantità escludano la loro presenza per mera evenienza statistica, e non siano presenti differenze non chiaramente giustificabili, si deve concludere che gli enti in questione sono eguali o hanno una stessa origine". ${ }^{1}$

1 Mastronardi, V., Trojani, A., (2014) "Appunti di Linguistica Forense - Introduzione e Reality Monitoring”. 
Scopo della linguistica forense è quello di attribuire la fonte del linguaggio ad un certo soggetto, di esaminare la veridicità di una testimonianza e di contribuire alla disciplina del profiling, ovvero della creazione di un profilo pricologico a partire da informazioni, in questo caso, appartenenti all'ambito linguistico.

\section{Memoria e processi cognitivi: elementi per la baseline}

La memoria si basa su di un processo dinamico, composto da fasi di percezione, codifica, immagazzinamento e recupero di dati. La codifica è fondata sull'assunto che elementi significativi abbiano un peso più rilevante, così come informazioni concrete ed eventi inusuali.

Pertanto la ricorstruzione di un ricordo non appare come un fenomeno statico, bensì complesso e fortemente attivo. Fattori di distorsione dell'informazione possono alterarne l'accuratezza e, di conseguenza, l'attendibilità. I suddetti fattori possono appartenere ad una di tre tipologie:

- Interni (propri dell'osservatore);

- Esterni (appartenenti ad informazioni successive all'evento);

- Relazionali (influenze nella comunicazione con l'intervistatore).

Risulta perciò fondamentale tenere conto di più variabili possibili nell'analisi di una testimonianza.

Estremamente utile a questo scopo è l'isolamento preliminare di un set di variabili che costituiscano la baseline, ovvero la base su cui poi verrà operata l'analisi della testimonianza. In particolare, è utile isolare caratteristiche quali l'età del soggetto, il suo livello di scolarizzazione, i tratti della sua personalità e le sue abitudini gestuali (per quanto riguarda l'osservazione del linguaggio non verbale). Inoltre, è importante considerare anche elementi come ansia o abilità nelle relazioni sociali e controllo personale. Quest'ultimo si riferisce al grado di spontaneità del soggetto, e al suo più o meno forte desiderio di apparire in un certo modo al proprio interlocutore (Vrij, Akehurst, Soukara \& Bull). Tenere in considerazione queste variabili è utile a discriminare quanto sia da attribuire alla natura del soggetto, da tutto ciò che invece sia opportuno osservare nella testimonianza.

Un racconto è tipicamente formato da:

- Contenuto della memoria;

- Contenuto dell'evento cui si è assistito;

- Processi cognitivi e decisionali su cui il soggetto intende o meno riferire". 2

Giuseppe Sartori, ordinario di neuropsicologia clinica e neuroscienze cognitive all'Università di Padova, ha identificato una lista di 26 criteri, da applicare per operare un controllo qualitativo su una data testimonianza. Questa check list risulta valida e completa, in quanto tiene conto di aspetti diversi ma ugualmente significativi. In particolare, si tratta di:

1. Distanza temporale tra fatto e prima rivelazione

L'accuratezza del ricordo appare inversamente proporzionale allo scorrere del tempo

2. Caratteristiche del fatto

Si distinguono fatti potenzialmente travisabili, da fatti non confondibili

3. Caratteristiche sensoriali del ricordo

2 Rossi, G., "L'intervista cognitiva in psicologia giuridica", Redazione Ops, https://www.opsonline.it/psychoinforma/lintervista-cognitiva-in-psicologia-giuridica/
Gli elementi sensoriali sono parte integrante di un ricordo. Sono particolarmente rilevanti per quando concerne le memorie flashbulb (memorie fotografiche).

4. Memoria traumatica

Un evento traumatico, associato ad un particolare stato emotivo, risulta solitamente più facile da ricordare rispetto ad un evento emotivamente neutro. (Kensinger e Shacter, 2006)

5. Ricordo di schemi (azioni ripetute)

Un'azione ripetuta nel tempo senza variazioni è detta script (copione). Il ricordo dell'azione nel suo complesso sarà nitido, a discapito di singoli dettagli, spesso omessi o costruiti.

6. Ripetizione del racconto

$\mathrm{Si}$ riferisce alla reiterazione della testimonianza. In questo caso, anche ricordi reali possono subire delle variazioni

7. Ricordo di eventi vissuti in prima persona o a cui si è assistito Azioni vissute personalmente sono ricordate con più efficacia rispetto ad eventi a cui si è assistito, che coinvolgono terze parti.

8. Contraddizioni interne al racconto

Contraddizioni, correzioni e incoerenze possono indicare: dimenticanza (un'informazione viene omessa), reminiscenza (una nuova informazione viene introdotta); incoerenza (le informazioni sono discordanti).

9. Arricchimento del ricordo (reminiscenza)

Dettagli vengono aggiunti in interviste o momenti successivi alla prima.

10. Oblio

Si riferisce alla possibilità per il soggetto di dimenticare, nel tempo, dettagli della narrazione.

11. Nucleo centrale e dettagli periferici

Il nucleo del racconto (chi, come, dove, quando, perché) tende a rimanere più stabile nel tempo. Al contrario, i dettagli periferici (colore, emozioni, sensazioni, ecc.) sono più suscettibili a variazioni.

12. Età del testimone

La percezione di un evento varia anche a seconda dell'età del soggetto, per quanto rigurada il focus durante l'esperienza, le conoscenze di partenza, i dettagli prevalenti. Uno stesso evento verrà descritto in modo differente da soggetti con età diverse.

13. Personalità del testimone

Le tendenze e le caratteristiche di personalità di un individuo ne influenzano il racconto. Un soggetto con tratti di personalità egoistica si concentrerà, ad esempio, sulle proprie sensazioni e i dettagli che lo riguardano personalmente.

14. Presenza di disabilità intellettiva/altra psicopatologia Disturbi e disabilità possono influire sui ricordi o sulla percezione della realtà.

15. Grado di sicurezza

Il grado di sicurezza nel racconto appare direttamente proporzionale all'accuratezza ed attendibilità dei dettagli.

16. Source monitoring

Si riferisce all'abilità di indivisuare la fonte (source) di un determinato ricordo (strumento utile a questo è il Reality Monitoring, trattato più avanti).

17. Motivazione

Si intende l'interesse specifico del soggetto a fornire la data testimonianza. Si distinguono, peraltro, motivazioni esterne (volte ad ottenere vantaggi) e interne (inconsapevoli).

18. Livello di riconoscibilità di facce in funzione della distanza (solo nel caso di riconoscimento di volti)

La riconoscibilità è inversamente proporzionale alla distanza dal volto.

19. Riconoscimento di persona sulla base della voce (solo nel caso di riconoscimento di persona)

Le probabilità sono variabili, ma solitamente al di sotto del $25 \%$. 


\section{Modalità di rivelazione del fatto}

La spontaneità del racconto ne aumenta l'accuratezza.

21. Domande suggestive

"Facilità con cui un individuo adotta ed inserisce nel proprio ricordo contenuti non veri che facevano invece parte delle domande ricevute." (Mazzoni, 2003)

22. Domande inaspettate

Domande insolite o particolari. In questo caso, la misurazione dei tempi di risposta risultà indicativa: il soggetto che ha intenzione di mentire impiegherà più tempo della formulazione di una risposta che ritiene valida.

23. Contagio dichiarativo

Avviene nel caso in cui più testimoni concordino su una determinata versione dei fatti o si confrontino sulle risposte fornite.

24. Assistenza psicologica/psicoterapica

E stato osservato come, a volte, riceve assistenza psicologica/psicoterapica prima di rendere testimonianza possa alterare i ricordi ed influenzarne l'attendibilità.

25. Effetto arma

È chiamato weapon-focus effect e si riferisce alla presenza, sulla scena raccontata, di un elemento di particolare impatto emotivo, come ad esempio l'arma impugnata da un criminale. L'attenzione del soggetto sarà focalizzata su tale elemento, ponendo in secondo piano altri dettagli.

26. Effetti di alcol e droga

La consumazione di alcol e droghe danneggia la memoria, rendendo la testimonianza meno attendibile. È interessante notare con l'effetto dell'alcol sui ricordi sia di entità maggiore rispetto a quello delle droghe.

L'analisi della testimonianza è mirata, soprattutto, all'identifica-

zione della menzogna, quindi alla discriminazione tra elementi reali ed elementi falsi. "La menzogna è anche indicata da (Gulotta, 2008):

- diminuzione: del numero di frasi in cui il soggetto afferma di aver avuto la possibilità di commettere reato, della qualità dei dettagli, della struttura logica, delle operazioni cognitive, della velocità dell'eloquio, del numero di frasi pronunciate, della riproduzione di conversazione, dei dettagli uditivi

- aumento: del tono di voce, del tono di voce lagnoso, del tempo di latenza, delle esitazioni dell'eloquio, del numero di frasi brevi, della descrizione di scambi interattivi, delle correzioni spontanee, dei dubbi circa la propria memoria, del numero delle informazioni irrilevanti, del numero delle dichiarazioni negative, degli errori nell'eloquio, del numero delle risposte indirette, del numero delle frasi con cui il soggetto prende distanza dal reato, del livello di gentilezza e condiscendenza, del numero di termini evasivi." 3

Al contrario, l'attendibilità di una testimonianza è essenzialmente valutabile attraverso due fattori onnicomprensivi (De Leo \& Biscione, 1999; Scali, Calabrese \& Biscione, 2003):

- accuratezza (rappresentazione corretta della realtà e riferimento della stessa)

- credibilità (motivazioni a fornire la testimonianza che possano influenzarne la veridicità).

\section{Il ruolo dell'intervistatore}

Le distorsioni della memoria possono, come precedentemente menzionato, anche dipendere da fattori relazionali e comunicativi. È per questo che il ruolo dell'intervistatore acquisisce ampia rilevanza, diventando di per sé una variabile di cui tenere conto. Suggerimenti, nuove informazioni e reiterazioni nelle domande possono influire largamente sulla testimonianza. Si definisce "effetto compiacenza", quando le stesse domande vengono ripetute più volte al soggetto, che adegua le proprie risposte in linea con ciò che crede l'intervistatore voglia sentire (Fornari, 2008). Il post-event misinformation effect, avviene invece quando nuove informazioni sono comunicate al soggetto dopo l'evento oggetto del racconto, con l'effetto di modificarne il ricordo a causa dell'aggiunta di quell'elemento all'interno della memoria.

Infine, la suggestionabilità, o suscettibilità, ad interrogatori coercitivi, viene studiata come il prodotto di: cedimento a domande suggestive (yelding) e cambio di atteggiamenti e risposte sotto pressione (shifting). Gudjonsson $(1992,2003)$ concretizza queste nozioni osservando:

- Il ricordo immediato che quantifica la capacità del soggetto di memorizzare, i livelli d'attenzione e concentrazione;

- Il ricordo differito che misura la capacità di rievocazione dopo un intervallo temporale di 50 minuti dall'esposizione all'evento;

- Yield 1 (Cedimento1), numero di suggestioni accettate dal soggetto nella prima intervista, prima del feedback negativo;

- Shift (Cambio), numero di volte che il soggetto modifica le risposte precedentemente fornite in seguito alla critica negativa dell'intervistatore;

- Yield 2 (Cedimento 2) numero di domande suggestive a cui il soggetto cede dopo il feedback negativo;

- La suggestionabilità totale punteggio ottenuto dalla somma tra il Cedimento 1 e il Cambio e si riferisce al grado generale di suggestionabilità di un individuo;

- La confabulazione. ${ }^{4}$

Correlati psicologici della suggestionabilità, come ansia, paura del giudizio altrui, desiderio di apprezzamento sociale, aspettative altrui, autostima ed assertività, contribuiscono, in misure e modi differenti, ad influire sul soggetto durante la testimonianza (Ceci, 1994).

Secondo una ricerca di Gulotta (2000), è fondamentale tenere conto del sentimento di vulnerabilità del soggetto interrogato, e della potenziale influenza che l'intervistatore, in virtù di questo, esercita. Viene evidenziato come il testimone si affidi al suo interlocutore, sia sensibile alla sua autorità e ritenga di doverne soddisfare le aspettative. Egli non desidera essere valutato negativamente, e la pressione esercitata dal contesto lo porta alla compiacenza.

È dunque fondamentale che l'intervistatore adotti un atteggiamento imparziale, che le sue parole non abbiano connotazioni emotive e che il colloquio sia condotto dal soggetto in modo spontaneo e libero. Domande aperte sono sicuramente preferibili a fornire opzioni che potrebbero suggerire al soggetto l'esistenza di una risposta "più corretta". Infatti, studi riportano come l'attendibilità di un discorso sia avvalorata quando la deposizione (Fornari, 2008; Fargnoli \& Moretti, 2005):

- È spontanea;

- Non è influenzata negativamente da condizioni particolari;

- Riguarda cose su cui l'interrogato non ha interesse a mentire;

- Presenta correlazioni con fatti noti;

- Concorda con altre deposizioni;

- Non presenta elementi contrastanti al proprio interno;

- Non presenta lacune, oblii o amnesie improvvise.

3 Castello, A., "Esame testimoniale: scoprire le menzogne tramite il contenuto verbale", in Psicologia giuridica, https://www. psicologiadellavoro.org/esame-testimoniale-scoprire-le-menzogne-tramite-il-contenuto-verbale/

4 Perretta, M.C., "Valutazione della suggestionabilità in ambito penale" (2018), Associazione Italiana di Psicologia Giuridica. 


\section{Reality Monitoring (RM)}

La pratica del Reality Monitoring ha come scopo quello di distinguere i ricordi di esperienze esterne, ovvero realmente vissute, da quelli di esperienze interne, cioè costruite, inventate.

Le memorie reali appaiono ricche di informazioni percettive, vale a dire di sfumature sensoriali quali odori, sapori, suoni, emozioni ed informazioni "contestuali" (movimenti e posizioni nello spazio). Al contrario, le memorie costruite sembrano mancare di questi elementi ed essere invece caratterizzate dalle cosiddette operazioni cognitive: pensieri, ragionamenti (es. "faceva freddo, quindi sicuramente avevo addosso una giacca pesante").

L'origine di questo strumento va ricercata negli studi sulla memoria condotti da M.L. Johnson e C.L. Raye (1981). Tenendo conto della nozione che i ricordi siano prodotti sia dai pensieri che da stimoli esterni, le loro ricerche si sono focalizzate su come le diverse fonti potessero produrre effetti sul ricordo stesso. L'origine esterna appare quindi fondata su processi percettivi, mentre quella interna si basa su immaginazione, ragionamento e processi costruttivi.

Risultato dello studio è una distinzione fra quattro diverse tipologie di informazione:

- Contestuali;

- Sensoriali;

- Semantiche;

- Cognitive.

Dove le prime tre tipologie sarebbero da riscontrare in memorie di tipo esterno, e l'ultima caratterizzerebbe ricordi interni al soggetto.

Studi successivi hanno poi ipotizzato che questa particolare distinzione potesse essere proiettata ulteriormente, per dimostrare il grado di veridicità di un racconto (Alonso-Quecuty, 1992).

Ai fini di stabilire l'entità di una data memoria, il Reality Monitornig si avvale di un set di otto criteri (Sporer,1997; Mastronardi, 2014):

- Chiarezza. Ovvero la chiarezza ed intensità della testimonianza. Il resoconto è chiaro, acuto e vivido?

- Informazioni percettive. È un criterio di valutazione della quantità e qualità delle esperienze sensoriali come suoni, odori, gusti, sensazioni fisiche e dettagli visivi;

- Informazioni spaziali. Descrizione spaziali, posizione di oggetti, persone;

- Informazioni temporali. La sequenza dei fatti, temporalmente espressa è esplicitamente descritta?

- Affetti. Le affermazioni contengono informazioni che descrivono lo stato emotivo durante i fatti descritti;

- Ricostruibilità della storia. Trattasi della possibilità di ricostruire gli eventi con i contenuti delle affermazioni fatte;

- Realismo. La storia è credibile? Realistica, ha un senso logico?

- Operazioni cognitive. Valutare la presenza di ragionamenti o inferenze fatte da altri attori dell'evento, durante l'evento.

Dai punteggi attribuiti alla soddisfazione dei sopracitati criteri si è potuto osservare come esperienze vissute realmente ottenessero risultati di gran lunga migliori, così come i racconti in prima persona. Inoltre, la variabile temporale si è scoperta estremamente rilevante: eventi più lontani nel tempo otterranno punteggi minori rispetto ad eventi più recenti (Sporer \& Kupper, 1995; Vrij et al., 2000; Sporer \& Sherman, 2006).

\section{Criteria Based Content Analysis (CBCA)}

La Criteria Based Content Analysis (CBCA), fra gli strumenti utilizzati per l'analisi della testimonianza, risulta il più completo, seppure non poggi su di un solido fondamento teorico. Differentemente dal Reality Monitoring, infatti, la CBCA non si fonda su teorie scientifiche, ma origina dall'osservazione pratica di casi studio.

La veridicità di un racconto o di una o più affermazioni è studiata attraverso un set di diciannove criteri, suddivisi in cinque macro-gruppi, che permettono di considerare elementi specifici della narrazione:

Caratteristiche generali (aspetti più generali della testimonianza nella sua globalità):

1. Struttura logica (congruenza e fondatezza);

2. Produzione non strutturata (la narrazione non è organizzata in maniera continua e l'informazione si presenta disseminata nel corso di essa);

3. Quantità dei dettagli (precisi elementi descrittivi riguardanti luogo, tempo, oggetti, persone e azioni);

Contenuto specifico (le diverse parti della deposizione vengono valutate nei termini di presenza e forza di determinati tipi di descrizioni):

4. Inserimento in un contesto (contestualizzazione e inserimento in una più ampia narrazione);

5. Descrizioni di interazioni (stretta connessione degli eventi riportati sia da un punto di vista verbale che comportamentale);

6. Riproduzione di conversazioni (discorsi, o parte di essi, nella loro forma originale);

7. Complicazioni inaspettate (episodi che possono pregiudicare il susseguirsi degli eventi);

Particolarità di contenuto (elementi che accrescono la realtà della narrazione):

8. Dettagli insoliti (elementi che risultano strani, atipici e inconsueti);

9. Dettagli superflui (informazioni che nonostante siano legate al contesto non risultano rilevanti per la descrizione dell'evento principale);

10. Dettagli fraintesi ma riportati correttamente (azioni ed eventi che il soggetto non capisce, ma il cui senso risulta chiaro all'intervistatore);

11. Associazioni esterne collegate (descrizioni di avvenimenti o discorsi legati in qualche modo all'argomento esposto, ma non verificatesi in quella circostanza);

12. Descrizione dello stato mentale soggettivo (racconto di emozioni, sentimenti e pensieri personali);

13. Attribuzione di uno stato mentale all'accusato (racconto di emozioni, sentimenti e pensieri dell'imputato);

Contenuto relativo alla motivazione ad accusare (elementi che riguardano la motivazione del soggetto a testimoniare):

14. Correzioni spontanee (chiarificazioni e precisazioni aumentano la credibilità della testimonianza e aiutano ad escludere l'ipotesi che il soggetto sia stato indotto a dichiarare il falso);

15. Ammissione di mancanza di memoria (dettagli mancanti nel racconto);

16. Emergere di dubbi sulla propria testimonianza (denota mancanza di interesse nel costruire una versione perfetta dei fatti);

17. Auto-deprecazione (la vittima sembra assumersi una parte di responsabilità di ciò che è avvenuto);

18. Perdono dell'accusato (dichiarazioni che spiegano o giustificano il comportamento dell'imputato);

Elementi specifici dell'offesa (descrizione del reato nei particolari, raramente presente nelle testimonianze credibili): 
19. Caratteristiche in dettaglio del reato;

Un punteggio viene poi calcolato, in base al racconto dell'esperienza da parte del soggetto (per un massimo di 38 punti):

- 0 se il criterio è assente;

- 1 se il criterio è presente;

- 2 se il criterio è fortemente presente

\section{Statement Validity Analysis (SVA)}

La Statement Validity Analysis trova le sue origini nel 1954, in Germania dell'Ovest; il suo utilizzo, studiato e operato da Undeutsch, era quello di determinare l'attendibilità di testimonianze di minori a proposito di abusi sessuali.

L'ipotesi su cui si basa questo tipo di analisi viene detta, appunto, ipotesi di Undeutsch, e consiste nell'assumere che un racconto reale differisca in contenuto e qualità da una deposizione prodotta dall'invenzione.

Partendo da questa ipotesi, Steller e Koehnken definirono la struttura della SVA come processo analitico formato da:

- Intervista semistrutturata: 1'operatore inizia ponendo domande generiche per testare le capacità del soggetto e costruire una baseline, per poi seguitare con quesiti sempre più specifici sul fatto oggetto di indagine.

- CBCA: la Criteria Based Content Analysis è utilizzata per osservare le caratteristiche del racconto e quantificarle producendo un punteggio.

- Check list di validità: un ultimo passaggio è operato per distinguere aspetti contestuali e motivazionali che possano aver prodotto effetti sulla testimonianza. La lista è composta da elementi inversamente proporzionali all'accuratezza ed attendibilità del colloquio (De Leo, Scali \& Caso, 2005):

- Caratteristiche psicologiche

- Linguaggio e conoscenze non appropriate

- Inadeguatezza delle emozioni

- Suggestionabilità

- Caratteristiche dell'intervista

- Domande suggestive, veicolanti o coercitive

- Inadeguatezza globale dell'intervista

- Motivazione

- Motivo per il quale è stata sporta la denuncia

- Motivazioni relative alle rivelazioni originali

- Pressione a rilasciare l'accusa

- Domande investigative

- Coerenza con l'ordine delle cose

- Coerenze con altri resoconti

- Coerenza con altre prove

Nonostante il primo studio sul campo (Esplin, Boychuk \& Raskin, 1988) sia stato condotto, anch'esso, a proposito di molestie su minori, la tecnica di analisi è riproducibile anche su soggetti adulti.

\section{Scientific Content Analysis (SCAN)}

L'Analisi del Contenuto Scientifico (SCAN) si presenta come uno strumento in grado di operare una distinzione tra affermazioni vere e costruite. Come le tecniche analizzate in precedenza, anche la SCAN si compone di criteri, sebbene non sia possibile individuarne una lista completa ed esauriente, né tantomeno un metodo ufficiale di assegnazione dei punteggi.

Si possono indicare come criteri presenti:

- Negazione

- Presentazioni sociali

- Struttura dell'affermazione
- Emozioni

- Tempi oggettivi e soggettivi

- Uso della lingua

- Cambiamenti nel registro linguistico

- Correzioni spontanee

- Mancanza di memoria

- Informazioni mancanti

La tecnica SCAN fa uso del VIEW (Verbal Inquiry Effective

Witness), un questionario formato da test con l'obiettivo specifico di indagare sulle affermazioni del soggetto.

\section{Step Wise Interview (SWI)}

La Step Wise Interview si propone come metodo ulteriore, in grado di disciplinare l'intervista e facilitarne l'esito positivo. Viene soprattutto impiegato nelle interazioni con soggetti minori, generalmente in casi di abusi o che coinvolgano molestie. Consta, pertanto, di 6 fasi:

- Costruzione del rapporto;

- Regole dell'intervista;

- Chiarire la necessità di dire la verità (facoltativo);

- Introdurre l'argomento di interesse;

- Fase di raccolta delle informazioni (narrazione libera, domande aperte, domande specifiche);

- Chiusura. $^{5}$

\section{Metodi a confronto}

È opportuno, a questo punto, operare un confronto tra le metodologie proposte, in particolare fra le più utilizzate: $\mathrm{RM}$ e CBCA/SVA.

Il Reality Monitoring risulta di utilizzo più lineare, sebbene i risultati appaiano meno precisi e maggiormente influenzabili da variabili esterne. La semplicità, nonostante ciò, è da ricercare nella distribuzione dei criteri osserbabili: non soltanto sono minori in numero, ma permettono di analizzare, allo stesso tempo e con la stessa precisione, sia il comportamento sincero, sia quello di menzogna, fornendo strumenti da ricercare in entrambe le condotte.

Inoltre, se il Reality Monitoring nasce e si sviluppa finalizzato all'analisi di soggetti adulti, il CBCA ha origine nell'osservazione di testimonianze di minori, ed appare pertanto di più congeniale applicazione in quell'ambito.

Sporer ha condotto uno studio a scopo comparativo, evidenziando gli elementi in comune alle due metodologie (Sporer, 1997):

- Consistenza logica e realismo: questo fattore è risultato legato positivamente anche alla ricostruibilità della storia (RM), mentre negativamente ai dettagli inusuali (CBCA);

- Quantità di dettagli e riferimenti contestuali: questo fattore include dettagli superficiali e inusuali (CBCA), ma anche le informazioni spaziali e temporali (RM);

- Pensieri ed emozioni: per quanto riguarda la CBCA questo fattore sembra essere legato al criterio che si riferisce ai riferimenti ai propri stati mentali mentre, in relazione ai criteri del

5 Yuille, J. C., Cooper, B.S., Hervé, H.F., (2009) "La nuova generazione delle Linee guida Step Wise per l'intervista dei minori, in Pedoparafilie: prospettive psicologiche, forensi, psichiatriche", Milano: Franco Angeli. 
$\mathrm{RM}$, sono risultate legate a questo fattore le operazioni cognitive e gli affetti;

- Chiarezza: questo fattore include il criterio della chiarezza $(\mathrm{RM})$, mentre risulta legato negativamente al criterio della produzione non strutturata (CBCA);

- Interazioni verbali e non verbali: questo fattore è caratterizzato dai criteri riproduzione di conversazioni e descrizione di interazioni, ma anche descrizione di stati mentali dell'accusato. Come si può vedere si tratta di criteri che appartengono esclusivamente alla CBCA, ma non può essere rilevato invece nessun collegamento con nessun criterio del RM (Sporer, 1997)." $"$

Ad entrambi i criteri, nonostante ciò, viene criticata una mancanza di approfondimento per quanto riguarda la definizione operativa dei criteri che li compongono (Tomasino, Carillo \& Grattagliano, 2008).

\section{Applicazioni pratiche: le chiamate ai soccorsi}

Uno studio condotto da T. Harpster e S. H. Adams negli Stati Uniti dimostra come l'analisi linguistico-forense sia applicabile anche a coloro che, dopo aver commesso un crimine, chiamano i soccorsi (in questo caso il 911).

L'analisi si propone, quindi, di individuare quelle chiamate al 911 da parte di soggetti che, dopo avere leso (mortalmente o non) la vittima, tentano di confondere le autorità, fingendo una richiesta d'aiuto. Un comportamento di questo tipo può essere identificato come uno step nel processo di staging, ovvero di alterazione della scena del crimine al fine di depistare le indagini.

Questo tipo di telefonata appare, però, unica e con caratteristiche ben diverse dalla classica chiamata di chi realmente sta chiedendo aiuto per una persona in difficoltà. Di seguito, gli elementi osservati (Harpster, Adams, 2017):

\section{Argomento della chiamata}

- Rilevanza delle informazioni: Spesso, i soggetti che chiamano il 911 per un tentativo di staging tendono ad aggiungere informazioni non richieste, poco utili ai fini del soccorso, con 1'obiettivo di perdere tempo e rallentare gli aiuti. Un chiamante innocente, al contrario, si affretta a dare le risposte necessarie senza aggiungere particolari irrilevanti.

- Comportamento nei confronti della vittima: Non sono rari, nei chiamanti colpevoli, insulti o espressioni denigratorie nei confronti della vittima, talvolta incolpandola per l'accaduto.

- Precisione nei fatti: Alcuni dei criminali analizzati cadevano in contraddizione o non avevano risposte precise per le domande dell'operatore, creando confusione e incomprensioni.

\section{Soggetto della chiamata}

- Argomento: I chiamanti innocenti tendono a focalizzare il dialogo sulla vittima e le sue condizioni. Al contrario, i colpevoli spostano il focus su sé stessi, facendosi protagonisti del problema.

- Focus dell'aiuto: Alcuni criminali, invece di chiedere soccorso per la vittima, hanno condotto la conversazione verso di sé, chiedendo aiuto per sé stessi e per il proprio stato emotivo.

- Comportamento nei confronti della morte della vittima: Uno

6 Tomasino, M.G., Carillo B.F., Grattagliano, I., "Statement Validity Analysis Reality Monitoring: analisi critica di due strumenti per valutare le affermazioni dei testimoni", Rassegna Italiana di Criminologia, anno II - n. 2, 2008. spettatore innocente non accetta la morte della vittima; chiede aiuto anche se le condizioni sono ormai disperate. È stato invece osservato come un colpevole abbia più facilità a dichiarare il decesso della vittima, anche quando questo non appare evidente.

\section{Modalità della chiamata}

- Modulazione della voce: La modulazione della voce è un segno di emotività, che spesso manca nel chiamante colpevole.

- Urgenza: Un chiamante innocente tenderà a richiedere aiuto in modo immediato, rapido, talvolta brusco e privo di convenevoli. Al contrario è stato analizzato come i criminali spesso aggiungano segmenti di conversazione superflui, come saluti, scuse o ringraziamenti. Inoltre, tendono a non utilizzare abbreviazioni o forme contratte. (questo vale per la lingua inglese)

- Cooperazione: La cooperazione è fondamentale per riceve aiuto in modo rapido e puntuale. Per questo un chiamante innocente tenderà a rispondere prontamente alle domande dell'operatore. Un criminale, contrariamente a ciò, eluderà le domande, ometterà dettagli o ripeterà frasi sconnesse, allungando i tempi di reazione dei soccorsi. Spesso, in questi casi, il chiamante non dispone di tutte le informazioni richieste dall'operatore, perché si trova nel processo di costruzione di una finta storia da presentare alle autorità. Per questo motivo non è raro che il soggetto in questione cambi idea nel bel mezzo di una frase, contraddicendosi o modificando la propria versione dei fatti.

Lo studio condotto da Harpster e Adams si presenta, dunque, come un ulteriore strumento in grado di esaminare il linguaggio e discriminare tra affermazioni o racconti sinceri e tentativi di inganno.

\section{Bibliografia}

Castello, A., Esame testimoniale: scoprire le menzogne tramite il contenuto verbale", in Psicologia giuridica. https://www.psicologiadellavoro.org/esame-testimoniale-scoprire-le-menzognetramite-il-contenuto-verbale/

Bogaard, G., Meijer, E.H., Vrji, A., Merckelbach, H., "Scientific Content Analysis (SCAN) Cannot Distinguish Between Truthful and Fabricated Accounts of a Negative Event", https://www. frontiersin.org/articles/10.3389/fpsyg.2016.00243/full

Gradante, F., (2019) "Il Metodo SVA con bambini vittime o testimoni di reati sessuali", ISFAR, https://www.isfarfirenze.it/formazione/metodo-sva-bambini/

Harpster, T., Adams, S.H. (2017) “Analyzing 911 Homicide Calls* - Practical aspects and applications"

Harpster, T., Adams, S.H. (2017) "Is the Caller the Killer? Analyzing 911 Homicide Calls.” Criminologia, anno II - n. 2, 2008.

LSI Laboratory for Scientific Interrogation, Inc., "The VIEW Questionnaire", https://www.lsiscan.com/id26.htm

Mastronardi, V., Trojani, A., (2014) "Appunti di Linguistica Forense - Introduzione e Reality Monitoring”.

Montemurro, M., "La rievocazione del ricordo nella testimonianza. Rassegna di studi", Università degli studi di Roma "La Sapienza".

Perretta, M.C., "Valutazione della suggestionabilità in ambito penale" (2018), Associazione Italiana di Psicologia Giuridica.

Rossi, G., "L'intervista cognitiva in psicologia giuridica", Redazione Ops, https://www.opsonline.it/psycho 
informa/lintervista-cognitiva-in-psicologia-giuridica/ Sartori, G., "Checklist sul livello qualitativo della testimonianza", https://www.testimonianzapenale.com/lista-argomenti/checklist-del-livello-qualitativo-della-testimonianza

Tomasino, M.G., Carillo B.F., Grattagliano, I., "Statement Validity Analysis Reality Monitoring: analisi critica di due strumenti per valutare le affermazioni dei testimoni”, Rassegna Italiana di Criminologia, anno II - n. 2, 2008.

Yuille, J.C., Cooper, B.S., Hervé, H.F., (2009) "La nuova generazione delle Linee guida Step Wise per l'intervista dei minori, in Pedoparafilie: prospettive psicologiche, forensi, psichiatriche", Milano: Franco Angeli.
Correspondence: Monica De Vincentis.

E-mail: devincentis.monica@gmail.com

Key words: Forensic linguistics; CBCA; reality monitoring. Parole chiave: Linguistica forense; $\mathrm{CBCA}$; reality monitoring. Palabras clave: Linguistica forense; $\mathrm{CBCA}$; reality monitoring.

Received for publication: 30 June 2020.

Accepted for publication: 3 August 2020.

This article is distributed under the terms of the Creative Commons Attribution Noncommercial License (by-nc 4.0) which permits any noncommercial use, distribution, and reproduction in any medium, provided the original author(s) and source are credited.

${ }^{\circ}$ Copyright: the Author(s), 2020

Licensee PAGEPress, Italy

Rivista di Psicopatologia Forense, Medicina Legale, Criminologia 2020; $25: 551$

doi:10.4081/psyco.2020.551 